\title{
Confinement and pseudoscalar glueball spectrum in the 2+1D QCD-like theory from the non-SUSY D2 brane
}

\author{
Adrita Chakraborty ${ }^{*}$ and Kuntal Nayek ${ }^{\dagger}$ \\ Indian Institute of Technology Kharagpur, Kharagpur 721302, India
}

(Received 3 August 2020; accepted 21 January 2021; published 1 March 2021)

\begin{abstract}
Here we study two important properties of $2+1$-dimensional QCD—confinement and pseudoscalar glueball spectrum - with holographic approach. We consider the low energy decoupled geometry of the isotropic non-supersymmetric D2 brane. We find the corresponding gauge theory is similar to the $2+1$ dimensional Yang-Mills (YM) theory with the running coupling $\lambda^{2}$. At the extremal limit (i.e., Bogomol'nyi-Prasad-Sommerfield limit), this gauge theory reduces to the super-YM theory. From the Nambu-Goto action of a test string, the potential of a $Q-\bar{Q}$ pair located on the boundary is calculated. At large $Q-\bar{Q}$ separation, it gives the tension $\sigma$ of the QCD flux tube. The parametric dependence of $\sigma$ is shown pictorially. It is found that $\sigma$ is a monotonically increasing function of the effective coupling $\lambda^{2}$. In comparison, $\sqrt{\sigma} / g_{\mathrm{YM}}^{2} N_{c}$ is found to match accurately with the previous results. In the next part, we consider fluctuation of the axion field in the aforementioned gravity background. From the linearized field equation of the fluctuation, we calculate the mass spectrum of $0^{-+}$numerically using the Wentzel-KramersBrillouin approximation. The pseudoscalar mass is found to be related to the string tension approximately as $M_{0^{-+}} / \sqrt{\sigma} \approx 3(n+2)$ for first three energy states, $n=0,1,2$.
\end{abstract}

DOI: $10.1103 /$ PhysRevD.103.066001

\section{INTRODUCTION}

According to our current understandings through various scattering experiments in RHIC, LHC, etc., most of the physical phenomena are mainly explained with the $3+1$-dimensional non-Abelian gauge theory-QCD. Since last five decades, the perturbative picture of this theory is almost well established with the proper theoretical models as well as the experimental evidences. There are some theoretical approaches like Massachusetts Institute of Technology (MIT) bag model, Skyrme model, chiral perturbation theory, heavy baryon perturbation theory for the strong coupling regime of QCD. However, this regime of the 3+1-dimensional QCD has not been explored enough compared to the perturbative regime. The lattice $\mathrm{QCD}$ has some nontrivial approach in this regime. Although the accuracy of the lattice calculation is restricted with various technical limitations and it is also unable to study the real-time dynamics of the theory. The lattice calculation with finite lattice spacing is approximated to the continuum limit

\footnotetext{
*adimanta09@iitkgp.ac.in

†kuntal.nayek@iitkgp.ac.in
}

Published by the American Physical Society under the terms of the Creative Commons Attribution 4.0 International license. Further distribution of this work must maintain attribution to the author(s) and the published article's title, journal citation, and DOI. Funded by SCOAP ${ }^{3}$. to map the real QCD. But this process is not always easy and accurate. Since last two decades, the string theory has become relevant to study the strongly coupled gauge theory through the holographic tool, called AdS/CFT correspondence $[1,2]$. In this correspondence, the nonperturbative regime of the supersymmetric Yang-Mills (YM) theory in $3+1$ dimension has been studied almost completely. But in reality our known QCD is not supersymmetric. So, still it is interesting to study the nonperturbative QCD in $3+1$ dimensions.

Addressing this issue, the nonperturbative $\mathrm{QCD}$ in lower dimension, e.g., 2+1-dimensional Yang-Mills theory has been studied $[3,4]$ due to the following reasons. Unlike the four-dimensional theory, it is comparatively simple to handle mathematically. However, unlike the fourdimensional theory, this theory having the dimension-full coupling $g_{\mathrm{YM}}^{2}$ is not classically scale invariant. In spite of this difference, the most important reason to study this lower dimensional theory is that there are many conceptual similarities with the $3+1$-dimensional Yang-Mills theory. The theory has ultraviolet freedom like four-dimensional theory. The dimensionless effective coupling of the theory $g_{\mathrm{YM}}^{2} \ell_{s}$ goes to zero at the ultrahigh energy scale, i.e., at the small length scale $\ell_{s} \rightarrow 0$. Also, the infrared slavery, confinement, and mass spectrum have the same characteristic as four-dimensional QCD. At low energy scale, the coupling becomes too large and both the theories are driven by self-coupling of the gluon. However, the 
$2+1$-dimensional Coulomb potential has weak logarithmic confinement $V_{C}(r) \sim g_{\mathrm{YM}}^{2} \log (r)$, the linear confinement can be found to have the well-known form at the nonperturbative regime, i.e., $V(r) \sim \sigma r$. In $2+1-$ dimensional pure Yang-Mills theory, the string tension of the QCD flux tube is theoretically [5] derived as

$$
\sigma=g_{\mathrm{YM}}^{4} \frac{N_{c}^{2}-1}{8 \pi}
$$

for large $N_{c}$. This direct proportionality of the binding energy with the spatial scale indicates the confined state of the bounded $Q-\bar{Q}$ pair. This confinement indicates the selfcoupling dominated nonperturbative regime. In this regime, due to the strong self-coupling, the free gluons form the bound states, called glueball. Like the four-dimensional theory, here the masses of the light glueball states are also found to be proportional to $\sqrt{\sigma}$. Along with these theoretical approaches, the three-dimensional YM theory has been studied in lattice simulation $[4,6,7]$. However, for the three-dimensional theory, the available data are not enough to get the complete information about the mass spectrum of the pseudoscalar glueball.

The AdS/CFT correspondence [1,2] states the duality between the supergravity theory in the $4+1$-dimensional anti-de Sitter (AdS) space-time and the $\mathcal{N}=4$ superYang-Mills theory in 3+1-dimensional flat space-time. According to this correspondence, a stack of $N_{c}$ Bogomol'nyi-Prasad-Sommerfield (BPS) D3 branes in the gravity theory is used to study the dual pure superYM theory. In the gravity theory, the string coupling $g_{s}$ is so small that the theory is perturbative. On the other hand, in the gauge theory, however the Yang-Mills coupling $g_{\mathrm{YM}}^{2}$ is small $\left(g_{\mathrm{YM}}^{2} \sim g_{s} \rightarrow 0\right)$, the number of the gauge fields $N_{c}$ is large, $N_{c} \rightarrow \infty$. The effective coupling of the gauge theory, $g_{\mathrm{YM}}^{2} N_{c}$, is finite and large enough to make the theory nonperturbative [1]. So, this duality is useful to study the nonperturbative quantum field theory with the large effective coupling through the holographic study of the perturbative gravity theory. Thus, it is also called strong/weak coupling duality. However, the AdS/CFT duality is restricted to the $\mathcal{N}=4$ super Yang-Mills theory on the AdS boundary, the same idea of the general gauge/gravity duality can be extended to the non-AdS gravity theory to study the strongly coupled Yang-Mills theory without the conformal symmetry [8]. In these holographic models, the effective gauge coupling is large which does not allow the theory to be asymptotically free [9]. Despite the absence of asymptotic freedom, many other nonperturbative behaviors of QCD like confinement, chiral symmetry breaking, scattering cross section, glueballs, thermal phase transition, etc., can be studied from holography $[8,10-16]$. The fourdimensional QCD has been also studied in the light-front holographic approach [17] and in lattice calculations [1821]. The general concept of gauge/gravity duality is also used in the lower dimensional branes where the dual theories are the lower dimensional QCD-like theories. In holographic approach, like four-dimensional gauge theory, the asymptotic freedom is not allowed in QCD3. But it allows to study other properties of QCD. The QCD3 has been already studied in holographic method $[2,12,22]$ by compactifying the D3 brane in one of its spatial directions to get the $3+1$-dimensional non-AdS gravity theory.

Here, in this paper, we intend to study three-dimensional QCD-like theory from the non-BPS D2 brane of the type-II supergravity. The confinement property and the mass spectrum of the pseudoscalar glueball are studied in this work. In ten-dimensional type-II supergravity theory, there is a set of nonsupersymmetric solutions [23] which does not follow the BPS condition. As the supersymmetry is broken, the solutions have more parameters than BPS solutions. In dual nonconformal gauge theory, these parameters are related to the coupling and the UV fixed point which make the theory QCD-like. Here we consider a stack of $N_{c}$ number of the non-supersymmetric (non-SUSY), isotropic D2 branes where the background dilaton field $\phi$ varies with the length scale of the bulk theory. According to gauge/ gravity duality, it corresponds to $2+1$-dimensional YangMills theory with running effective coupling which is analogous to $2+1$-dimensional QCD. The low energy decoupling limit is considered to find the near brane geometry of the non-SUSY D2 brane. Then we configure a probe string whose end points (representing the $Q-\bar{Q}$ pair on $t-x^{1}$ plane) are separated by a large distance $\Delta x$ along one of the spatial directions of the brane $x^{1}$. Following the holographic dictionary, the thermal expectation value of the timelike Wilson loop $W^{F}(\mathcal{C})$ is computed from the minimal world sheet area swept out by the test string on the boundary surface of the gravity theory which coincides with the loop $\mathcal{C}[2,10,24]$. Hence, we use the following relation (2) to find the $Q-\bar{Q}$ potential energy $V(\Delta x)$ from the minimal Nambu-Goto action $S(\mathcal{C})$ :

$$
W^{F}(\mathcal{C})=\operatorname{Exp}[i S(\mathcal{C})]=\operatorname{Exp}[i V(\mathcal{C}) \mathcal{T}],
$$

where $\mathcal{T}$ is the length of the temporal direction of the loop $\mathcal{C}$. For large separation, the $Q-\bar{Q}$ potential is found to be linearly proportional to the separation, $V(\Delta x) \propto \Delta x$ which ensures the confinement nature of the gauge theory. In the holographic approach, the mass spectrum can be evaluated from the fluctuations in the gravity theory. According to the properties of the fluctuated fields, we get different types of glueballs, e.g., scalar, vector, and tensor fields, respectively, which correspond to the spin-0, spin-1, and higher spin glueballs. The naked singularity of the non-SUSY D2 does not allow us to find the scalar glueball with the particular method used herein. However, this singularity should not be an issue if we approach it with some other method. So here we study the pseudoscalar glueball only. To study this glueball, we consider the 
fluctuation of the axion field in the same decoupled background. As the axion is the minimally coupled scalar, the fluctuation does not perturb the background metric. From the potential energy term in the Schrödinger-like field equation of the fluctuation, we compute the mass spectrum of the pseudoscalar glueball. The mass spectrum is evaluated numerically using the Wentzel-Kramers-Brillouin (WKB) approximation. The restrictions in the WKB method bound us in the lower states only. In the nonperturbative regime, since the effective coupling is very large, the theory is dominated by the self-coupling of the gluon fields. So, in this regime, we can compare our results with the Yang-Mills theory without the flavor quark. The pseudoscalar glueball has spin zero, odd parity, and even charge parity, $0^{-+}$. So far, the estimated ground state mass of the pseudoscalar glueball $0^{-+}$is $2590 \mathrm{MeV}$ [18] and $M_{-+}^{*} / M_{-+}=1.46$ [2] in $3+1$ dimensions. In four dimensions, the glueball mass is found to increase with the gauge coupling. The same feature is expected in lower dimension too.

This paper is arranged as follows. In Sec. II, we discuss the non-SUSY D2 brane and its decoupling limit. After that in the following section, the confinement is studied from the $Q-\bar{Q}$ potential using a test string. Then the mass spectrum is calculated and the result is compared with the known data in Sec. IV. Finally, in the last section, we conclude the study.

\section{GRAVITY BACKGROUND}

In this section, we will discuss the non-SUSY D2 brane solution and its decoupled geometry at low energy limit.

\section{A. Non-SUSY D2 brane}

Besides the supersymmetric BPS brane, there is a similar sector of the nonsupersymmetric solutions of the type-II supergravity, which are called, in short, non-SUSY $\mathrm{D} p$ brane [23]. Unlike the BPS $\mathrm{D} p$ brane, these are nonextremal solutions, i.e., the ADM mass and charge are not equal rather follow the gravitational censorship. Due to this nonequality, the non-SUSY branes are characterized by more parameters. In the BPS limit, which is indeed the extremal limit, the number of free parameters reduces and the non-SUSY branes merge into BPS branes of same dimensions. At low energy limit, the non-SUSY branes decouple from the bulk theory in similar manner as BPS branes do $[25,26]$. But, in these cases, the decoupled geometries are not AdS or conformal to AdS. It means the decoupled geometry is not symmetric under the conformal transformations. According to the gauge/gravity duality, this non-AdS gravity theory corresponds to the nonconformal gauge theory defined in the world volume of the brane. Again, the dilaton field associated to this non-SUSY brane is found to be nontrivial and depends on the length scale of the gravity theory. So, in holographic dictionary, the dual gauge theories in case of these non-SUSY D $p$ branes are similar to the nonsupersymetric Yang-Mills theory with the running coupling.

The non-SUSY D2 brane solution can be derived by putting $p=2$ and rearranging the harmonic function $F(\rho)$ in (2.11)-(2.12) of [25]

$$
\begin{aligned}
d s^{2}= & F(\rho)^{-\frac{1}{2}}, G(\rho)^{\frac{\beta}{4}+\frac{\delta}{4}}\left(-d t^{2}+\sum_{i=1}^{2}\left(d x^{i}\right)^{2}\right) \\
& +F(\rho)^{\frac{1}{2}}, G(\rho)^{\frac{1}{5}-\frac{\beta}{4}+\frac{\delta}{4}}\left(\frac{d \rho^{2}}{G(\rho)}+\rho^{2} d \Omega_{6}^{2}\right) \\
e^{2 \phi}= & e^{2 \phi_{0}} F(\rho)^{\frac{1}{2}}, G(\rho)^{\delta-\frac{\beta}{4}}, \quad F_{[6]}=Q \operatorname{Vol}\left(\Omega_{6}\right),
\end{aligned}
$$

where the harmonic functions are

$$
\begin{aligned}
& F(\rho)=G(\rho)^{\gamma} \cosh ^{2} \theta-\sinh ^{2} \theta \\
& G(\rho)=1+\frac{\rho_{2}^{5}}{\rho^{5}} .
\end{aligned}
$$

Here the background is given in the string frame. The $(2+1)$-dimensional world volume is defined with the coordinates $\left(t, x^{1}, x^{2}\right)$, whereas the seven-dimensional transverse space is defined by the spherical coordinates $\left(\rho, \Omega_{6}\right) . \rho$ is the radial coordinate perpendicular to the world volume, i.e., the brane is located at $\rho=0$. Therefore, the geometry has a singularity at $\rho=0$. The dilaton field $\phi$ is the function of radial coordinate. The boundary expectation value of dilaton, $\phi_{0}$, is related to the string coupling $g_{s}=e^{\phi_{0}}$. As the effective coupling of the theory depends on the dilaton field, it also varies with the length scale $\rho$ of the theory or the energy scale $(\sim 1 / \rho)$. $\rho_{2}$ is a constant point on the length scale, also known as the mass parameter. $\theta$ is a dimensionless constant, known as the charge parameter, related to the total Ramond-Ramond (RR) charge $Q$ of the brane. Along with these three, there are three more dimensionless parameters $-\delta, \beta$, and $\gamma$. These six parameters are mutually related via the following three relations derived from [25]

$$
\begin{aligned}
\beta & =\gamma+\frac{\delta}{4} \\
\gamma & =\sqrt{\frac{12}{5}-\frac{15}{16} \delta^{2}} \\
Q & =\frac{5}{2} \gamma \rho_{2}^{5} \sinh 2 \theta .
\end{aligned}
$$

Therefore, out of the six we have only three independent free parameters $-\rho_{2}, \theta$, and $\delta$. Now, according to the definition, the harmonic function $G(\rho)$ is always greater than 1 . Validity of the metric in the range $0<\rho<\infty$ refers $F(\rho)$ to be positive, which suggests that $\gamma$ must be positive 
and real. So, the value of the parameter $\delta$ is bounded in the range $-\frac{8}{5} \leq \delta \leq \frac{8}{5}$.

In the BPS limit [23], which is eventually the extremal limit, $\rho_{2} \rightarrow 0$ and $\theta \rightarrow \infty$, but $Q$ remains finite and reduces to the RR charge of the BPS D2 brane; $Q=5 R_{2}^{5}$, where $R_{2}$ is the mass parameter of the BPS D2 brane. Therefore, in this limit, $G(\rho) \sim 1$ and $F(\rho)$ reduce to the harmonic function of BPS D2 brane, i.e., $F(\rho) \rightarrow 1+\frac{R_{2}^{5}}{\rho^{5}}$. Thus, the background (3) reduces to the BPS D2 brane.

\section{B. Decoupled geometry}

Now, to study the gauge/gravity duality in this nonSUSY background, at the low energy limit, we must have two completely decoupled theories - theory with gravity and theory without gravity. The theory without gravity, i.e., the gauge theory lives on the brane. This decoupling occurs when the gravitational excitations living in the bulk decouple from the theory on the brane. This has been already confirmed in previous studies from the graviton scattering cross section [25]. The graviton scattering in this background at low energy limit shows that the near brane regime decouples from the ten-dimensional bulk. Here we are interested in the near brane decoupled geometry of nonSUSY D2 brane. As the fundamental string length $\ell_{s} \rightarrow 0$ at the low energy limit, we consider the length scale of the bulk accordingly to get a finite scale in near brane regime. Now, following the decoupled geometry of the non-SUSY D3 brane [27], we scale the radial coordinate and parameters of (3) in the following way:

$$
\rho=\alpha^{\prime} u, \quad \rho_{2}=\alpha^{\prime} u_{2}, \quad \sinh ^{2} \theta=\frac{L}{\gamma u_{2}^{5} \alpha^{\prime 2}},
$$

where $\alpha^{\prime}=\ell_{s}^{2}$ has mass dimension -2 . Since $\rho$ has mass dimension $-1, u$ has mass dimension $+1 . u$ is taken as the radial coordinate of the decoupled geometry which is identified as the energy scale of the theory. $u_{2}$ is a constant parameter on the energy scale $u$, which is the cause of the nonconformal structure of the theory. The other quantity $L$ is defined as $L=3 \pi^{2} g_{\mathrm{YM}}^{2} N_{c}$, where $g_{\mathrm{YM}}^{2}$ is the Yang-Mills coupling in 2+1-dimensional Yang-Mills theory and $N_{c}$ is the color charges. In gravity theory, Yang-Mills coupling is related to the string coupling $g_{\mathrm{YM}}^{2}=2 g_{s} \alpha^{\prime-\frac{1}{2}}$, and $N_{c}$ is the number of the $\mathrm{D} 2$ branes. As $g_{s}$ is dimensionless, the YangMills coupling has mass dimension 1 . As $\alpha^{\prime} \rightarrow 0$, the string coupling $g_{s}$ has to be very small too so that the dilaton field does not diverge near the singularity $\rho=0$. This also makes the gauge coupling small enough. To satisfy the gauge/ gravity duality, as $g_{\mathrm{YM}}^{2}$ is small, $N_{c}$ must be very large. Thus, using the scaling (6), we can write the decoupled geometry of the background (3) as follows:

$$
\begin{aligned}
d s^{2} & =\alpha^{\prime} G_{\mu \nu} d X^{\mu} d X^{\nu} \\
& =\alpha^{\prime}\left[\sqrt{\frac{\gamma u_{2}^{5}}{L}} F(u)^{-\frac{1}{2}}, G(u)^{\frac{\delta}{4}+\frac{\beta}{4}}\left(-d t^{2}+\sum_{i=1}^{2}\left(d x^{i}\right)^{2}\right)+\sqrt{\frac{L}{\gamma u_{2}^{5}}} F(u)^{\frac{1}{2}}, G(u)^{\frac{1}{5}+\frac{\delta}{4}-\frac{\beta}{4}}\left(\frac{d u^{2}}{G(u)}+u^{2} d \Omega_{6}^{2}\right)\right] \\
e^{2 \phi} & =\frac{g_{\mathrm{YM}}^{4}}{4} \sqrt{\frac{L}{\gamma u_{2}^{5}}} F(u)^{\frac{1}{2}}, G(u)^{\delta-\frac{\beta}{4}} \\
F_{[6]} & =5 L \alpha^{\prime 3} \operatorname{vol}\left(\Omega_{6}\right),
\end{aligned}
$$

where the harmonic functions get the new form under the decoupling limit (6) as

$$
F(u)=G(u)^{\gamma}-1, \quad G(u)=1+\frac{u_{2}^{5}}{u^{5}} .
$$

In these decoupled forms of the harmonic functions, $\gamma$ cannot be zero. So the allowed range of $\delta$ is now modified to $-\frac{8}{5}<\delta<\frac{8}{5}$. This decoupled geometry is non-AdS; there is no conformal symmetry. Now, as we have considered $L$ to be very large, the curvature of the six-dimensional transverse sphere is small enough at the finite energy scale which validates the application of the gauge/gravity duality. In (7), the corresponding $(2+1)$-dimensional gauge theory on the boundary is expected to be nonconformal theory due to the presence of $u_{2}$ in the gravity theory. As we take $u_{2} \rightarrow 0$, the metric becomes AdS with a nontrivial conformal factor same as we get it from BPS D2 brane at low energy limit. So $u_{2}$ is related to the fixed energy scale in the corresponding gauge theory. Now the effective gauge coupling $\lambda^{2}$ can be written from the dilaton field following the standard relation $e^{\phi} \sim \frac{\lambda^{\frac{5}{2}}}{N_{c}}[2]$,

$$
\lambda^{2}=\frac{1}{\left(6 \pi^{2}\right)^{\frac{4}{5}} \gamma^{\frac{1}{5}}}\left(\frac{L}{u_{2}}\right) F(u)^{\frac{1}{5}} G(u)^{\frac{2}{\delta} \delta-\frac{\beta}{10}},
$$

which varies with the energy scale $u$ and the parameter $\delta$. Thus, in the corresponding gauge theory, we have the running coupling similar to QCD. So, the theory on the brane is a $(2+1)$-dimensional QCD-like theory without flavor. Since the Yang-Mills coupling is directly proportional to $g_{s}$ and inversely proportional to the fundamental 


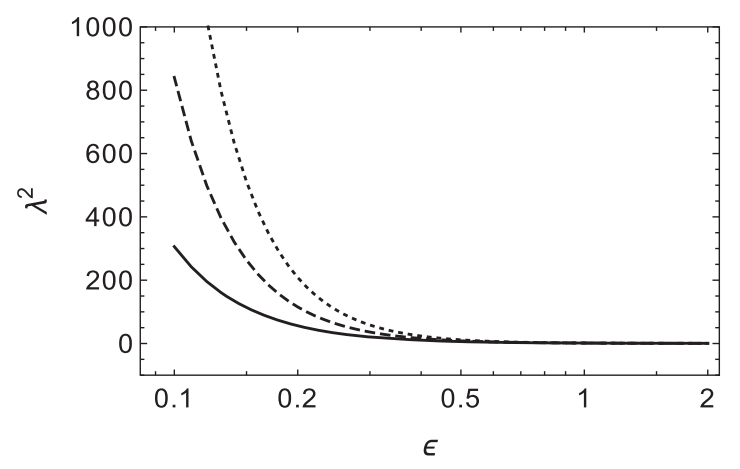

FIG. 1. Dependence of $\lambda^{2}$ on the energy scale $u=\epsilon u_{2}$ for $\delta=0.98$ (solid line), 1.28 (dashed line), and 1.58 (dotted line) at $u_{2}=g_{\mathrm{YM}}^{2} N_{c}$.

string length, at low energy limit in supergravity theory it remains finite and nonzero which does not allow the theory to be asymptotically free. Like the QCD theory, in (9), the effective gauge coupling $\lambda^{2}$ decays with the increasing energy scale $u=\epsilon u_{2}$ which is shown in Fig. 1. Here we have plotted $\lambda^{2}$ versus $u$ for three different $\delta$ values, $\delta=0.98,1.28,1.58$. $\lambda$ is found to have large value at small $u$ and diverges at the singularity $u=0$. Near the singularity $\lambda^{2} \gg 1$ which is the self-coupling dominated regime. In the asymptotic region $u \rightarrow \infty$, the coupling constant goes to zero mathematically. But, in the holographic study, the $\lambda<1$ regime is not allowed. At the fixed energy scale $u, \lambda^{2}$ is also found to increase monotonically with the increasing value of $\delta$.

Now, at this point, we can understand the significance of the dimension-full parameter $u_{2}$ from the behavior of the theory on the various energy scales $u$. At $u \gg u_{2}$, the geometry (7) reduces to the decoupled geometry of the BPS D2 brane and the effective coupling becomes

$$
\lambda^{2} \approx \frac{\left(6 \pi^{2}\right)^{\frac{1}{5}}}{2} \frac{g_{\mathrm{YM}}^{2} N_{c}}{u} \sim \frac{g_{\mathrm{YM}}^{2} N_{c}}{u} .
$$

As $\frac{\left(6 \pi^{2}\right)^{\frac{1}{5}}}{2}=1.1$, we can consider it as 1 . The corresponding gauge theory for the BPS D2 brane has been discussed in details in [2]. In this case, gauge theory is perturbative if

$$
u \gg g_{\mathrm{YM}}^{2} N_{c} .
$$

On the other hand, at the limit $u \ll u_{2}$, the effective coupling $\lambda^{2}$ becomes very large for the given range of $\delta$. In this regime, the effective string coupling $e^{\phi} \gg 1$ when

$$
u^{\frac{\gamma}{2}+\frac{15}{8} \delta} \ll \frac{1}{\gamma^{1 / 5}} g_{\mathrm{YM}}^{2} N_{c}^{\frac{1}{5}} u_{2}^{\frac{\gamma}{2}+\frac{15}{8} \delta-1} .
$$

Then we need to uplift the non-SUSY D2 brane to the non-SUSY M2 brane solution defined in 11 dimensions. Therefore, the world volume theory of the non-SUSY D2 brane is holographically dual to the type IIA supergravity in the range $u^{\frac{\gamma}{2}+\frac{15}{8} \delta}>\frac{1}{\gamma^{1 / 5}} g_{\mathrm{YM}}^{2} N_{c}^{\frac{1}{5}} u_{2}^{\frac{\gamma}{2}+\frac{15}{8} \delta-1}$ and $u<g_{\mathrm{YM}}^{2} N_{c}$. Now the 2+1-dimensional Yang-Mills theory has only one dimension-full parameter which is the coupling $g_{\mathrm{YM}}^{2}$. So, in this picture, the bulk parameter $u_{2}$ is related to the coupling $g_{\mathrm{YM}}^{2}$. Now combining $u \gg u_{2}$ with (11), $u_{2}$ can have a maximum value $g_{\mathrm{YM}}^{2} N_{c}$. Again, from the limits $u \ll u_{2}$ and (12), $u_{2}$ can have a lower limit $\frac{1}{\gamma^{1 / 5}} g_{\mathrm{YM}}^{2} N_{c}^{\frac{1}{5}}$. So, according to holography, the type-IIA supergravity describes the nonperturbative gauge theory for the above range of $u$, only if

$$
\frac{1}{\gamma^{1 / 5}} g_{\mathrm{YM}}^{2} N_{c}^{\frac{1}{5}} \leq u_{2} \leq g_{\mathrm{YM}}^{2} N_{c} .
$$

Since from the above analysis, the maximum value of $u$ can be order of $g_{\mathrm{YM}}^{2} N_{c}$, if we take $u_{2} \ll g_{\mathrm{YM}}^{2} N_{c}$, such upper limit of $u$ results $u \gg u_{2}$ which leads the theory to the world volume theory of the BPS D2 brane. So, for $u_{2}=\frac{1}{\gamma^{1 / 5}} g_{\mathrm{YM}}^{2} N_{c}^{\frac{1}{5}}$, we get the world volume theory of BPS D2 brane for the whole range of $u$. Here we take $u_{2}=g_{\mathrm{YM}}^{2} N_{c}$. This choice ensures that we are entirely in the nonperturbative world volume theory of the non-SUSY D2 brane for the above range of $u$. In other words, we do our calculations in nonperturbative regime near the perturbative boundary.

\section{CONFINEMENT}

Now to study the confinement property we need to find the binding potential of a largely separated static $Q-\bar{Q}$ pair. To do that, we consider a quark-antiquark pair on the boundary of the gravity background and an open string connecting the pair is hanging toward the singularity $u=0$ in the bulk. The two-dimensional world sheet swept by this open string (test string) is compared with the Wilson loop in the flat boundary metric which gives the $Q-\bar{Q}$ potential using (2). The area of the Wilson loop is given by the Nambu-Goto action, in the decoupled geometry, which can be written as follows:

$$
S=\frac{1}{2 \pi} \int d^{2} \sigma \sqrt{-\operatorname{Det}\left(g_{\alpha \beta}\right)} .
$$

The action is calculated on two-dimensional world sheet governed by the coordinates $\left(\sigma^{0}, \sigma^{1}\right)$. The metric $g_{\alpha \beta}$ is the pull-back of the bulk geometry on the world sheet. This two-dimensional pull-back metric is $g_{\alpha \beta}=G_{\mu \nu} \frac{\partial X^{\mu}}{\partial \sigma^{\alpha}} \frac{\partial X^{\nu}}{\partial \sigma^{\beta}}$, where $X^{\mu}$ and $\sigma^{\alpha}$ are, respectively, bulk and world sheet coordinates. Here we choose the test string with the following parametrization:

$$
\sigma^{0} \equiv t, \quad \sigma^{1} \equiv x^{1}=x(\text { say }), \quad u=u(x),
$$


where the other bulk coordinates remain localized at constant values. $x$ denotes the $Q-\bar{Q}$ separation which depends on the vertical length of the test string inside the bulk. With this parametrization, we derive the twodimensional worldsheet metric,

$$
\begin{aligned}
& g_{\sigma^{0} \sigma^{0}}=G_{t t} \\
& g_{\sigma^{0} \sigma^{1}}=g_{\sigma^{1} \sigma^{0}}=0 \\
& g_{\sigma^{1} \sigma^{1}}=G_{x^{1} x^{1}}+G_{u u}\left(\frac{d u}{d x}\right)^{2} .
\end{aligned}
$$

Therefore, the action integral (14) takes the following form:

$$
S=\frac{1}{2 \pi} \int d t d x P(u)\left[1+M(u)^{2}\left(\frac{d u}{d x}\right)^{2}\right]^{\frac{1}{2}},
$$

where

$$
\begin{aligned}
& P(u)=\sqrt{-G_{t t} G_{x^{1} x^{1}}}=\sqrt{\frac{\gamma u_{2}^{5}}{L}} F(u)^{-\frac{1}{2}} \cdot G(u)^{\frac{\delta}{4}+\frac{\beta}{4}}, \\
& M(u)=\sqrt{G_{u u} G^{x^{1} x^{1}}}=\sqrt{\frac{L}{\gamma u_{2}^{5}} F(u)^{\frac{1}{2}}, G(u)^{-\frac{2}{5}-\frac{\beta}{4}} .}
\end{aligned}
$$

The action depends on the slope of $u(x)$ - the evolution of the string inside the bulk, $d u / d x$, i.e., the rate of change of the distance $u$ of the string from the singularity with the $Q-\bar{Q}$ separation $x$. This slope vanishes at the stable configuration (stable position of the turning point) of the string. Now, as the $Q-\bar{Q}$ separation increases, the turning point moves toward the singularity, i.e., $u$ decreases. So, the position of the turning point is minimum at the maximum separation. First, we go to the stable point of the string by setting $d u / d x=0$, then we find the lowest position of that point taking the global minimum of $P(u)$. In this configuration, the maximum $Q-\bar{Q}$ separation is found to have the turning point at $u=u_{m}$,

$$
u_{m}=\frac{u_{2}}{\left(\Sigma^{1 / \gamma}-1\right)^{1 / 5}} \quad \text { where, } \Sigma=\frac{5 \delta+4 \gamma}{5 \delta-4 \gamma} .
$$

It is clear from the above turning point that it does not exist for the whole parametric range. Since $\gamma$ is always positive, it exists only if $\Sigma>1$ or $5 \delta+4 \gamma>5 \delta-4 \gamma$. Then the allowed parametric regime is now $\frac{2 \sqrt{6}}{5}<\delta<\frac{8}{5}$. Beyond this range, the test string goes into the singularity without any valid turning point. The variation of the ratio $\frac{u_{m}}{u_{2}}$ with the parameter $\delta$ in this specified parametric range is depicted in Fig. 2. Now from the Wilson loop equation (2), the binding energy or the potential is related to the area of the loop as $V=S / \mathcal{T}$, where $\mathcal{T}$ is the length of the temporal side of the loop $\mathcal{C}$, i.e., time runs from 0 to $\mathcal{T}$. Thus, the binding potential of the $Q-\bar{Q}$ pair at the maximum separation $\Delta x$ (i.e., at $u=u_{m}$ ) is

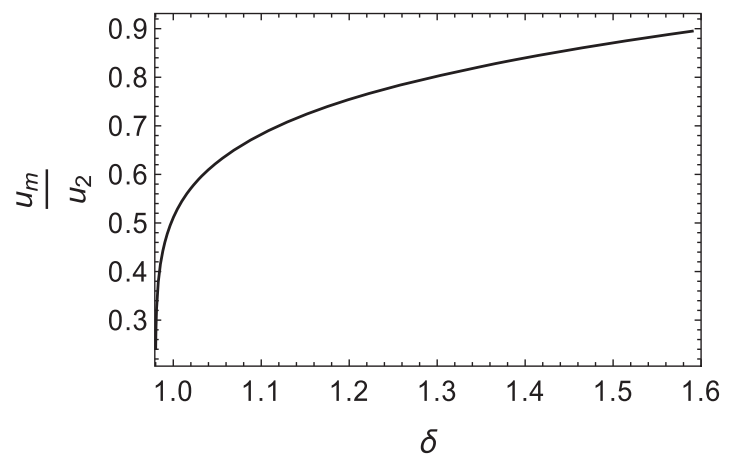

FIG. 2. Dependence of the ratio $\frac{u_{m}}{u_{2}}$ with $\delta$.

$$
\begin{aligned}
V & =\frac{1}{2 \pi} \int d x P\left(u_{m}\right) \\
& =\frac{u_{2}^{\frac{5}{2}}}{2 \pi \sqrt{L}}\left[\frac{5}{8} \delta^{2}-\frac{3}{5}\right]^{\frac{1}{4}} \Sigma^{\frac{5 \delta}{16 \gamma}} \Delta x,
\end{aligned}
$$

where $\int d x=\Delta x$ is the length of the flux tube. In (20), the $Q-\bar{Q}$ potential is linearly proportional to this spatial length. Coefficient of $\Delta x$ has dimension of string tension, which is identified as the QCD string tension $\sigma$. From (20), we can extract $\sigma$ as

$$
\frac{\sigma}{g_{\mathrm{YM}}^{4} N_{c}^{2}}=\frac{9 \pi^{3}}{2}\left(\frac{u_{2}}{L}\right)^{\frac{5}{2}}\left(\frac{5}{8} \delta^{2}-\frac{3}{5}\right)^{\frac{1}{4}} \Sigma^{\frac{5 \delta}{16 \gamma}}
$$

Here in $(2+1)$-dimensional theory, the flux-tube tension depends on the fixed energy scale $u_{2}$, the dimension-full Yang-Mills coupling $g_{\mathrm{YM}}^{2} N_{c}$, and on the background parameter $\delta$. Again, the effective gauge coupling $\lambda^{2}$ at $u=u_{m}$ also depends on these quantities,

$$
\lambda^{2}\left(u_{m}\right)=\frac{1}{\left(6 \pi^{2}\right)^{\frac{4}{5}}}\left(\frac{L}{u_{2}}\right)\left[\frac{5}{8} \delta^{2}-\frac{3}{5}\right]^{-\frac{1}{10}} \Sigma^{\frac{3 \delta}{8 \gamma}} .
$$

However, the $\delta$ dependence profile of $\lambda^{2}\left(u_{m}\right)$ is not similar as $\lambda^{2}(u)$. For a fixed $u, \lambda^{2}(u)$ is a monotonically increasing function of $\delta$ and for a fixed $\delta$, it is a decreasing function of $u$. We have also seen $u_{m}$ increases with the increasing value of $\delta$. So, $\lambda^{2}\left(u_{m}\right)$ varies with the combined effect of these two. As a result of this dependence, $\lambda^{2}\left(u_{m}\right)$ is found to decrease with $\delta$. Since $\lambda^{2}\left(u_{m}\right)$ is a dimensionless constant and $u_{2}$ has the same dimension as $L$, so in (21), $\sigma$ is dimensionally proportional to $g_{\mathrm{YM}}^{4} N_{c}^{2}$, i.e., $\sqrt{\sigma}$ has dimension of mass. Now we replace $u_{2}$ in (21) and rewrite the expression in terms of the dimensionless quantity $\lambda\left(u_{m}\right)$,

$$
\sigma=\frac{\left(g_{\mathrm{YM}}^{2} N_{c}\right)^{2}}{8 \pi \lambda^{5}\left(u_{m}\right)} \Sigma^{\frac{5 \delta}{4 \gamma}}=\frac{1}{2 \pi \alpha^{\prime}} \frac{\left(g_{s} N_{c}\right)^{2}}{\lambda^{5}\left(u_{m}\right)} \Sigma^{\frac{5 \delta}{4 \gamma}} .
$$

So, the dependence of $\sigma$ as $\sqrt{\sigma} \propto g_{\mathrm{YM}}^{2} N_{c}$ is similar to (1) which validates the expression in (23). Since $\alpha^{\prime} \rightarrow 0$ 
TABLE I. Values of $\sqrt{\sigma}$ calculated from (23) at $u_{2}=g_{\mathrm{YM}}^{2} N_{c}$.

\begin{tabular}{cccccccc}
\hline \hline$\delta$ & 0.98 & 1.08 & 1.18 & 1.28 & 1.38 & 1.48 & 1.58 \\
\hline$\frac{\sqrt{\sigma}}{g_{\mathrm{YM}_{N} N_{c}}}$ & 0.1799 & 0.1912 & 0.1984 & 0.2044 & 0.2097 & 0.2144 & 0.2187 \\
\hline \hline
\end{tabular}

because of decoupling limit and $g_{s} \rightarrow 0$ to get the perturbative gravity theory, the ratio $g_{s} / \alpha^{\prime}$ is finite. Again, as the gauge theory is strongly coupled, $\lambda$ is large, but the ratio $N_{c}^{2} / \lambda^{5}$ is finite due to large $N_{c}$. Therefore, the QCD string tension has a finite value. $\delta$ dependence of $\sigma$ is given in Table I. Here we have taken $u_{2}=g_{\mathrm{YM}}^{2} N_{c}$ which makes the ratio $\frac{\sqrt{\sigma}}{g_{\mathrm{YM}}^{2} N_{c}}$ independent of $g_{\mathrm{YM}}^{2} N_{c}$. Now, the theoretical value of $\sqrt{\sigma}$ is $0.1995^{1}$ for pure Yang-Mills theory. In lattice calculation [4], the value is 0.1975 . Both of these values are evaluated at $N_{c}=\infty$. Here for various $\delta, \sqrt{\sigma} / g_{\mathrm{YM}}^{2} N_{c}$ varies in the range $0.19 \pm 0.02$ for large $N_{c}$. So, our calculation fairly matches with those previous results. It is also clear from Table I that the string tension is a monotonically increasing function of $\delta$ which is depicted in Fig. 3. As we have seen previously, $\lambda^{2}$ increases with $\delta$ and the string tension increases with the increasing effective coupling $\lambda^{2}$. This dependence of the string tension on the effective coupling strengthens the QCD-like nature of the string tension calculated herein.

In the BPS limit, since $u_{2} \rightarrow 0$, the gravity background (7) reduces to the decoupled geometry of the BPS D2 brane and the dual gauge theory becomes $\mathcal{N}=8$ super-YangMills theory in $2+1$ dimensions. Since the super-YM theory is not a confined theory, the string tension $\sigma$ does not exist there. This can be shown here too. In our case, for a moment, assume $\sigma$ exists. Now, if we take $u_{2} \rightarrow 0$, to get a finite turning point in (19), we need to take $\Sigma \rightarrow 1$, i.e., $\gamma \rightarrow 0$ or $\delta \rightarrow \frac{8}{5}$. Now, as $\delta \rightarrow \frac{8}{5}$, the string tension in (21) takes the form $\frac{\sigma}{g_{\mathrm{YM}}^{4} N_{c}^{2}}=\frac{9 \pi^{3}}{2}\left(\frac{u_{2}}{L}\right)^{\frac{5}{2}}$, which goes to zero as $u_{2} \rightarrow 0$. So, in the BPS limit, the theory becomes deconfined like the $(2+1)$-dimensional $\mathcal{N}=8$ super-YangMills theory.

The analytic expression (23) shows that the flux tube tension increases with the increasing value of $\lambda$ and $g_{\mathrm{YM}}^{2}$ at large $N_{c}$. So the binding energy of the $Q-\bar{Q}$ pair bounded by the flux tube also increases with these couplings, which is consistent with the expected gauge theory. The linear nature of the potential in (20) indicates that the $Q-\bar{Q}$ binding energy increases with the increase of their separation. This is the confinement property of

\footnotetext{
${ }^{1}$ At $N_{c} \rightarrow \infty$, one can expand (1) as

$$
\frac{\sqrt{\sigma}}{g_{\mathrm{YM}}^{2} N_{c}}=\frac{1}{\sqrt{8 \pi}}\left(1-\frac{1}{2 N_{c}^{2}}+\frac{1}{8 N_{c}^{4}}+\cdots\right) .
$$
}

So, at $N_{c}=\infty, \sqrt{\sigma} / g_{\mathrm{YM}}^{2} N_{c}=\frac{1}{\sqrt{8 \pi}}=0.1995$.

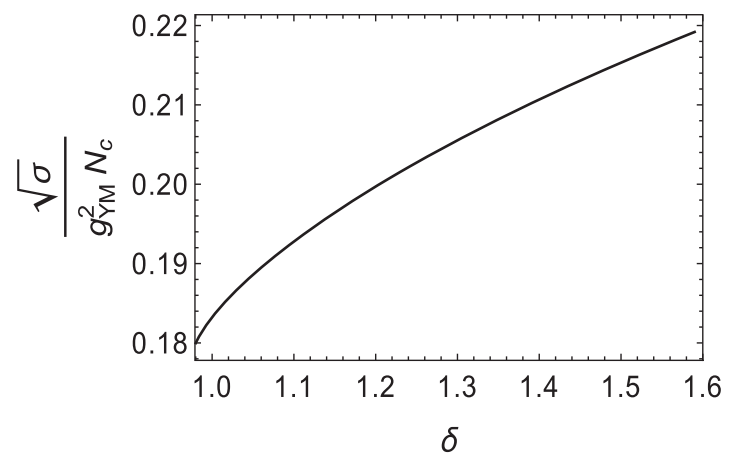

FIG. 3. $\delta$ dependence of QCD string tension $\sigma$ at $u_{2}=g_{\mathrm{YM}}^{2} N_{c}$.

the QCD. The existence of the confinement indicates the theory to be in the self-coupling dominated nonperturbative regime.

\section{MASS SPECTRUM OF $0^{-+}$}

In this self-coupling dominated regime, the confinement nature of the theory brings the gluons closer and makes the bound states-glueball. Though there is no complete theoretical model of the nonperturbative strongly coupled gauge theory, an empirical model of the glueball in QCD4 has been constructed in the Bag model $[28,29]$. Although the glueball is not observed experimentally till date, the mass spectrum of the glueballs has been calculated theoretically $[10,14,30]$ and in lattice QCD [4,18,31]. Those discrete spectra have also been found in the holographic QCD approaches. The same study leads us to the glueballs in the three-dimensional QCD—both in lattice [4,19,32] and holographic approaches [2,5,22]. Here we study the pseudoscalar glueball spectrum in holographic QCD in three dimensions using the non-SUSY solutions of the type-II supergravity. The pseudoscalar glueball mass is associated with the axion field of the bulk.

In our bulk theory (3), the axion field is zero. But one can consider the fluctuation of this axion to be nonzero. As the axion field couples minimally with the background, the fluctuation does not change the background metric. Now considering the action for the minimally coupled scalar, the linearized field equation for axion fluctuation $\chi$ can be written as

$$
\frac{1}{\sqrt{-G}} \partial_{\mu}\left(\sqrt{-G} G^{\mu \nu} \partial_{\nu}\right) \chi=0
$$

where $G$ is the determinant of the background metric $G_{\mu \nu}$ (7) and $\mu, \nu$ run over all of the ten coordinates of the decoupled geometry. Now, for simplicity, we demand the fluctuation field $\chi$ to be symmetric on the six-dimensional transverse sphere of (7), polarized along the brane world volume, and also a function of $u$. So we take $\chi=$ $f(u) e^{i k_{a} x^{a}}$, where $a=0,1,2$ and $k_{a} k^{a}=-M^{2} . M$ denotes 
the mass of the pseudoscalar glueball $0^{-+}$. Along with these assumptions using the geometry (7), we get from (24),

$$
\begin{aligned}
\partial_{u}^{2} f & +\left[\frac{6}{u}+\frac{1}{2}, \frac{\partial_{u} F}{F}+\left(1+\delta-\frac{\beta}{4}\right) \frac{\partial_{u} G}{G}\right] \partial_{u} f \\
+ & \frac{L M^{2}}{\gamma u_{2}^{5}} F G^{-\frac{4}{5}-\frac{\beta}{2}} f=0 .
\end{aligned}
$$

As the harmonic functions change very rapidly near the singularity, we take the coordinate transformation $u=u_{2} e^{y}$ to properly analyze the whole range of $u$. $y$ is the new dimensionless radial coordinate. The radial coordinate range is now zoomed into $-\infty<y<\infty$ from $0<u<\infty$. Using this new coordinate, the above equation can be written as follows:

$$
\begin{aligned}
\partial_{y}^{2} f & +\left[5+\frac{1}{2}, \frac{\partial_{y} F}{F}+\left(1+\delta-\frac{\beta}{4}\right) \frac{\partial_{y} G}{G}\right] \partial_{y} f \\
+ & \frac{L M^{2}}{\gamma u_{2}^{3}} e^{2 y} F G^{-\frac{4}{5}-\frac{\beta}{2}} f=0 .
\end{aligned}
$$

Substituting $f(y)=e^{-\frac{5}{2} y} F^{-\frac{1}{4}} G^{-\frac{1}{2},-\frac{\delta}{2}+\frac{\beta}{8}} \kappa(y)$ yields the standard form of the differential equation to use the WKB method,

$$
\partial_{y}^{2} \kappa(y)-V(y) \kappa(y)=0 .
$$

This is a second order Schrödinger-like wave equation. The potential function is given as

$$
\begin{aligned}
V(y)= & \frac{25}{4}+\frac{25}{4} \frac{\left(\frac{15}{16} \delta-\frac{1}{4} \gamma+1\right)\left(\frac{15}{16} \delta-\frac{1}{4} \gamma-1\right)}{\left(e^{5 y}+1\right)^{2}} \\
& +\frac{25}{4} \frac{\gamma\left(\frac{15}{16} \delta+\frac{3}{4} \gamma\right)}{\left(e^{5 y}+1\right)^{2}\left(1-\left(1+e^{-5 y}\right)^{-\gamma}\right)} \\
& -\frac{75}{16} \frac{\gamma^{2}}{\left(e^{5 y}+1\right)^{2}\left(1-\left(1+e^{-5 y}\right)^{-\gamma}\right)^{2}} \\
& -\frac{m^{2}}{\gamma} e^{2 y} \frac{\left(1+e^{-5 y}\right)^{\gamma}-1}{\left(1+e^{-5 y}\right)^{\frac{4}{5}+\frac{\gamma}{2}+\frac{\delta}{8}}},
\end{aligned}
$$

where the mass $M$ is made dimensionless quantity $m$ by using $m^{2}=M^{2} L / u_{2}^{3}$. To analyze the nature of the potential function, we consider the asymptotic expansions. The approximated expansion of $V(y)$ at $y \rightarrow \infty$ is

$$
V(y) \approx \frac{25}{4}-m^{2} e^{-3 y} .
$$

So, the potential function gradually reaches to the constant numerical value $25 / 4$ at positive infinity and the crossover point in this direction is at $y_{+}=\frac{2}{3} \ln \left(\frac{2}{5} m\right)$. At the negative asymptote, $y \rightarrow-\infty$, the potential function can be approximated as

$$
V(y) \approx \frac{25}{4}\left(\frac{15}{16} \delta+\frac{1}{4} \gamma\right)^{2}-\frac{m^{2}}{\gamma} e^{\left(6-\frac{5}{2} \gamma+\frac{5}{8} \delta\right) y} .
$$

Here also $V(y)$ merges with a positive constant $\frac{25}{4}\left(\frac{15}{16} \delta+\frac{1}{4} \gamma\right)^{2}$ with a crossover at $y_{-}=\frac{16}{48-20 \gamma+5 \delta} \ln \left(\frac{5}{2} \frac{\sqrt{\gamma}}{m}\left(\frac{15}{16} \delta+\frac{1}{4} \gamma\right)\right)$. So, the potential is almost flat and positive in whole range except $y_{-} \leq y \leq y_{+}$. Thus, it forms a small potential well with boundaries at $y_{-}$and $y_{+}$. The depth of the well is regulated by $\delta$ and the mass $m$. Thus, using WKB approximation, we can estimate $m$ for given $\delta$. The WKB approximation equation is

$$
\int_{y_{-}}^{y_{+}} d y \sqrt{-V(y)}=\left(n+\frac{1}{2}\right) \pi,
$$

where $n=0,1, \ldots$ denote various energy states. The integrand in (31) is function of $\delta$ and $m$. So, solving this equation, $m$ can be evaluated for the given $\delta$ and $n$. This approximation is valid if the depth of the well is small enough. As the depth of the well is directly proportional to the mass $m$ (which can be shown easily by plotting $V$ for different values of $m$ ), the WKB approximation is a good approximation for the low energy states. Here we calculate first few energy states of the spectrum. Using numerical tools, we solve (31) and find the values of $M$ in the unit of $\sqrt{u_{2}^{3} / L}$. Then we numerically calculate the ratio $M / \sqrt{\sigma}$ and compare them with some previous results.

For the scalar glueball $0^{++}$, the Schrödinger-like wave equation can be found easily from the linearized equation of the dilaton field in the Einstein frame. In this case, the potential well is not bounded at the negative $y$ regime, i.e., $y_{-} \rightarrow-\infty$. Now, as the background geometry (7) has singularity at $y=-\infty$, we need to put a cutoff near the singularity to evaluate the spectrum using WKB approximation (31). Although, the spectrum is then found to depend on the cutoff. Again, the gravity theory near the singularity has the large value of the effective coupling $\lambda^{2}$ and therefore, the theory has to be uplifted to the 11dimensional theory. So, to find the scalar glueball spectrum in this present background, the WKB approximation method does not work.

Since the analytic turning points $y_{+}$and $y_{-}$come from the asymptotic expansions ignoring higher order terms, the actual turning points for the whole potential $V(y)$ slightly differ from those values. So, in numerical method, first we find those exact turning points numerically. Then using those values, Eq. (31) is numerically solved for $m$. In Table II, we have listed the dimensionless scaled mass $m$ for different $\delta$, where the actual mass $M_{0^{-+}}$is $m \sqrt{u_{2}^{3} / L}$. The mass values have been found to increase with the increasing values of $\delta$. We have already seen the effective coupling $\lambda$ as an increasing function of $\delta$. So, the increasing of $\delta$ causes increase in the self-coupling of the gluon fields. Due to this rising of self-coupling, the glueballs become 
TABLE II. Here we calculate the mass $M_{0^{-+}}$and $\sqrt{\sigma}$ in unit of $\sqrt{u_{2}^{3} / L}$ and $10^{-3} L$, respectively, for various $d$, where $M_{0^{-+}}$, $M_{0^{-+}}^{*}$, and $M_{0^{-+}}^{* *}$ denote the mass of the ground state, first excited state, and second excited state, respectively.

\begin{tabular}{lcccc}
\hline \hline$\delta$ & $M_{0^{-+}}$ & $M_{0^{-+}}^{*}$ & $M_{0^{*+}}^{* *}$ & $\sqrt{\sigma^{\mathrm{a}}}$ \\
\hline 0.98 & 6.15797 & 9.70152 & 12.9685 & 6.07865 \\
1.08 & 6.25563 & 9.86602 & 13.2028 & 6.45687 \\
1.18 & 6.35031 & 10.0252 & 13.4294 & 6.70183 \\
1.28 & 6.44245 & 10.1799 & 13.6493 & 6.90446 \\
1.38 & 6.53266 & 10.3310 & 13.8638 & 7.08193 \\
1.48 & 6.62202 & 10.4803 & 14.0746 & 7.24190 \\
1.58 & 6.71494 & 10.6346 & 14.2905 & 7.38863 \\
\hline \hline
\end{tabular}

${ }^{\mathrm{a}}$ These values have been found by dividing the values in Table I by $3 \pi^{2}$.

more massive in the sea of gluon. Therefore, in this aspect, the calculated mass value shows the consistent nature. The ratio $M_{0^{-+}}^{*} / M_{0^{-+}}$are 1.575 and 1.583 for $\delta=0.98$ and $\delta=1.58$, respectively. We can also evaluate the ratio $M_{0^{-+}} / \sqrt{\sigma}$. To do that, we first write $M$ in the unit of $L$ same as $\sqrt{\sigma}$ using $u_{2}=L /\left(3 \pi^{2}\right)$. From Table II, these ratios are roughly equal to 6,9 , and 12 for the ground state $(n=0)$, first excited state $(n=1)$, and second excited state $(n=2)$, respectively. Thus, using an empirical relation for $u_{2}=g_{\mathrm{YM}}^{2} N_{c}$, we can relate the pseudoscalar glueball mass and the QCD string tension as follows:

$$
M_{0^{-+}} \approx \frac{10^{3}}{6 \sqrt{3} \pi^{3}}(n+2) \sqrt{\sigma} \approx 3(n+2) \sqrt{\sigma},
$$

where $n=0,1$, and 2. In (32), the first "approximation" sign is used as we are writing this relation for the few states not the whole spectrum and the second approximation sign is used simply because of the numerical approximation. Therefore, the mass-to-tension ratio is almost constant for a particular energy state, which is one of the similarities of QCD3 with the four-dimensional QCD. From this empirical relation, the ratio of the mass of the first excited state to that of the ground state mass is 1.5 . To date, we have not found the enough data for the full pseudoscalar spectrum. But the complete spectrum is available in four dimensions. So now, we first take a comparative look on those results. In QCD4 [18], $M_{0^{++}}^{*} / M_{0^{++}}=1.54$ and $M_{0^{-+}} / M_{0^{++}}=1.50$. On the other hand, in QCD3, these two ratios are, respectively, 1.5061 and 2.148 in lattice calculation [4]. However, in holographic approach [2,12], $M_{0^{++}}^{*} / M_{0^{++}}=$ 1.72 in QCD3. So, it seems that the glueballs are comparatively more massive in $2+1$ dimensions. Therefore, we can expect the ratio $M_{0^{-+}}^{*} / M_{0^{-+}}$which is found to be 1.40 in lattice calculation [18] and 1.46 in holography [12] for QCD4 acquires a higher value in the $2+1$-dimensional theory. Here we get the ratio in the range 1.575-1.583. Due to the behavioral consistencies of this mass spectrum with the QCD, we can expect that these masses are also consistent with the pure Yang-Mills theory in $2+1$ dimensions.

\section{CONCLUSION}

To conclude, in this paper, we have obtained the string tension and the pseudoscalar glueball spectrum in $2+1$ dimensional strongly coupled gauge theory from the nonSUSY D2 brane of type-IIA supergravity. The gravity background has two free parameters-the dimensionless $\delta$ and the dimension-full $u_{2} . u_{2}$ controls the nature of the gauge theory and $\delta$ measures the zero temperature gluon condensate in that gauge theory. Here we have taken $u_{2} \sim g_{\mathrm{YM}}^{2} N_{c}$, which ensures the corresponding gauge theory is strongly coupled $2+1$-dimensional Yang-Mills theory, i.e., the 2+1-dimensional QCD. Here we would like to mention that in case of the non-SUSY brane the world volume theory may contain some open string tachyonic field $[33,34]$. But, as we have observed that the decoupled gravity background (7), asymptotically reduce to the near horizon geometry of the BPS D2 brane, i.e., this near horizon geometry is some deformation of the near horizon geometry of the BPS D2 brane, so our corresponding gauge theory is some perturbation of the world volume theory of the BPS D2 brane $[23,25]$. Therefore, the gauge theory considered here is a stable $2+1$-dimensional Yang-Mills theory without tachyon. ${ }^{2}$ In Table I, the string tension $\sigma$ is found to depend on the parameter $\delta$. This parametric dependence has been shown pictorially in Fig. 3. It can be found that for a particular value of $\delta$, the string tension matches exactly with the results of the pure Yang-Mills theory in $2+1$ dimension. In this paper, we have numerically calculated the mass spectrum of the pseudoscalar glueball using WKB approximation. The spectrum given in Table II is found to have consistent nature with QCD. The masses increase with the increasing effective coupling of the theory. However, the coupling dependence of the spectra has not been shown explicitly. The ratio of the mass to the square root of the string tension has been found to be constant which is analogous to the four-dimensional QCD.

Here in this study the non-AdS form of the decoupled geometry (7) of the non-SUSY D2 brane indicates that the holographic gauge theory is the nonsupersymmetric

\footnotetext{
${ }^{2}$ In the background (3), when $\theta=0$, the charge of the nonSUSY D2 brane becomes zero. Then the world volume theory becomes fully tachyonic. In general, for finite $\theta$, the world volume theory is the Yang-Mills theory in the presence of tachyonic field. It can be shown that for large $\theta$ and $\gamma=1$ the background reduces to a deformation of the near horizon geometry of BPS D2 brane which is tachyon free. Again, the background (3) becomes BPS D2 brane in the BPS limit. So, in this case, the decoupling limit (6) makes the charge parameter $\theta$ large, which ensures the absence of the tachyon in the world volume theory.
} 
Yang-Mills theory. The effective gauge coupling $\lambda^{2}$ is monotonically decreasing function of the energy scale $u$ which is shown in Fig. 1. We have also seen the string tension and the pseudoscalar glueball spectrum show the consistent behaviors. Also, the existence of the parameter $\delta$ ensures the gluon condensate in dual gauge theory. All of these similarities indicate that the holographic dual of the non-SUSY D2 brane in the considered parametric range is the 2+1-dimensional QCD-like theory. But the non-AdS geometrical structure of the gravity background has made it hard to find the complete picture of this QCD-like gauge theory.

Due to the complicated mathematical structure of the background, we have evaluated the mass spectrum numerically using WKB approximation. It will be interesting to find the mass spectrum analytically. In this way, we will be able to find the analytic expressions of the masses showing explicit dependencies on various parameters. In analytic method, the singularity will no longer be an issue in case of the scalar glueballs. Therefore, as an extension of this we can find the analytic expression of the scalar and also the higher spin glueballs in this background. The thermal evolution of the string tension and the glueball spectrum in the $2+1$ dimensional gauge theory is also an interesting topic to study in non-SUSY brane background of type-II supergravity.

\section{ACKNOWLEDGMENTS}

We want to acknowledge Shibaji Roy and Kamal L Panigrahi for some important discussions to execute this problem. K. N. wants to acknowledge the Department of Physics, IIT Kharagpur for the funding.
[1] J. M. Maldacena, Int. J. Theor. Phys. 38, 1113 (1999).

[2] O. Aharony, S. S. Gubser, J. M. Maldacena, H. Ooguri, and Y. Oz, Phys. Rep. 323, 183 (2000).

[3] D. Karabali and V. P. Nair, Nucl. Phys. B464, 135 (1996); Phys. Lett. B 379, 141 (1996); Int. J. Mod. Phys. A 12, 1161 (1997); D. Karabali, C. j. Kim, and V. P. Nair, Phys. Lett. B 434, 103 (1998).

[4] M. J. Teper, Phys. Rev. D 59, 014512 (1998).

[5] V. P. Nair, Nucl. Phys. B, Proc. Suppl. 108, 194 (2002).

[6] M. Teper, Phys. Lett. B 311, 223 (1993).

[7] O. Philipsen, M. Teper, and H. Wittig, Nucl. Phys. B469, 445 (1996).

[8] E. Witten, Adv. Theor. Math. Phys. 2, 505 (1998).

[9] J. Polchinski and M. J. Strassler, Phys. Rev. Lett. 88, 031601 (2002).

[10] N. R. Constable and R. C. Myers, J. High Energy Phys. 11 (1999) 020.

[11] H. Ooguri, H. Robins, and J. Tannenhauser, Phys. Lett. B 437, 77 (1998).

[12] C. Csaki, H. Ooguri, Y. Oz, and J. Terning, J. High Energy Phys. 01 (1999) 017.

[13] J. Babington, J. Erdmenger, N. J. Evans, Z. Guralnik, and I. Kirsch, Phys. Rev. D 69, 066007 (2004).

[14] C. Csaki and M. Reece, J. High Energy Phys. 05 (2007) 062.

[15] Y. Kim, B. H. Lee, C. Park, and S. J. Sin, J. High Energy Phys. 09 (2007) 105.

[16] J. Polchinski and M. J. Strassler, J. High Energy Phys. 05 (2003) 012.

[17] S. J. Brodsky, G. F. de Teramond, and A. Deur, Phys. Rev. D 81, 096010 (2010); G. F. de Teramond and S. J. Brodsky, Nucl. Phys. B, Proc. Suppl. 199, 89 (2010).
[18] C. J. Morningstar and M. J. Peardon, Phys. Rev. D 60, 034509 (1999).

[19] M. Teper, Phys. Lett. B 397, 223 (1997).

[20] D. E. Miller, Phys. Rep. 443, 55 (2007).

[21] B. Lucini and M. Teper, J. High Energy Phys. 06 (2001) 050 .

[22] D. K. Hong and H. U. Yee, J. High Energy Phys. 05 (2010) 036.

[23] J. X. Lu and S. Roy, J. High Energy Phys. 02 (2005) 001.

[24] H. Liu, K. Rajagopal, and U. A. Wiedemann, J. High Energy Phys. 03 (2007) 066.

[25] K. Nayek and S. Roy, J. High Energy Phys. 03 (2016) 102.

[26] S. Roy, arXiv:1705.00329.

[27] K. Nayek and S. Roy, Phys. Lett. B 766, 192 (2017).

[28] A. Chodos, R. L. Jaffe, K. Johnson, C. B. Thorn, and V. F. Weisskopf, Phys. Rev. D 9, 3471 (1974).

[29] A. Chodos, R. L. Jaffe, K. Johnson, and C. B. Thorn, Phys. Rev. D 10, 2599 (1974).

[30] R. de Mello Koch, A. Jevicki, M. Mihailescu, and J. P. Nunes, Phys. Rev. D 58, 105009 (1998).

[31] Y. Chen, A. Alexandru, S. J. Dong, T. Draper, I. Horvath, F. X. Lee, K. F. Liu, N. Mathur, C. Morningstar, M. Peardon, S. Tamhankar, B. L. Young, and J. B. Zhang, Phys. Rev. D 73, 014516 (2006).

[32] O. Philipsen, M. Teper, and H. Wittig, Nucl. Phys. B, Proc. Suppl. 53, 626 (1997).

[33] A. Sen, Classical Quantum Gravity 17, 1251 (2000).

[34] A. Sen, Int. J. Mod. Phys. A 20, 5513 (2005). 